\title{
Resonant Light Coupling to Microspheres using Gaussian Beams of Optical Fibers
}

\author{
Ali Serpengüzel, ${ }^{*}$ Temel Bilici, ${ }^{\dagger}$ Şenol İşçi, ${ }^{\dagger}$ and Adnan Kurt \\ Koç University, Microphotonics Research Laboratory, Physics Department, \\ Rumeli Feneri Yolu, Sariyer, Istanbul 34450 Turkey
}

\begin{abstract}
Morphology dependent resonances of dielectric microspheres are used for polarization insensitive optical channel dropping from an optical fiber half coupler to a silicon photodetector in the M-band. The dropped channels are observed in the elastic scattering and the transmission spectra. The highest quality factor morphology dependent resonances have a repetitive channel separation of $0.14 \mathrm{~nm}$ and a linewidth of $0.06 \mathrm{~nm}$. The filter drops approximately $10 \%(0.5 \mathrm{~dB})$ of the power at the resonance wavelength. The power detected by the photodiode is estimated to be approximately $3.5 \%$ of the power in the fiber.
\end{abstract}

Keywords: Channel dropping filter, integrated optoelectronics, microsphere resonator, morphology dependent resonances, whispering gallery modes.

\section{INTRODUCTION}

In recent years, dielectric microspheres ( $\mu$-spheres) have found various applications in photonics and optoelectronics ${ }^{1}$. Morphology dependent resonances (MDR's) or whispering gallery modes (WGM's) of dielectric $\mu$-spheres provide the necessary optical feedback for applications in spectroscopy, laser science and optical communications, such as new kinds of microlasers, optical couplers, and optical filters ${ }^{2}$. Low threshold lasing from Nd-doped silica $\mu$-spheres 2 , polymer $\mu$-sphere lasers ${ }^{3}$ and Raman lasers have been demonstrated. Strain tunable $\mu$-sphere oscillators ${ }^{4}$, add-drop filters ${ }^{5}$, and thermooptical switching 6 have been realized for frequency control in optical communications for filtering, multiplexing, and switching.

Microsphere resonators are uniquely applicable in compact optoelectronic devices for wavelength division multiplexing (WDM) applications ${ }^{7}$. In optical communication, WDM is important for increasing the bandwidth of the current fiber optic networks. In WDM, the final optical to electronic conversion needs an all-optical packet-switching layer, which consists of all-optical gates, interferometers, semiconductor optical amplifiers, resonant cavity enhanced photodetectors, optical random access memory elements, and channel dropping filters. In these planar lightwave circuits, dielectric $\mu-$ spheres with their MDR's, can be used as compact channel dropping filters.

In this paper, we report for the first time to our knowledge the channel dropping of light in an optical fiber using a dielectric microsphere integrated with a silicon photodetector. The detection of MDR's is performed by using a windowless wide area silicon photodiode in proximity to the $\mu$-sphere, which is placed on an optical fiber half coupler (OFHC). The MDR's in the elastic scattering spectrum and associated dips in the transmission spectrum are experimentally observed. This coupling geometry is suitable for the manufacturing of polarization-independent integrated optoelectronic devices for optical communications.

* aserpenguzel@ku.edu.tr; phone: + 90 (212) 338-1312; fax: + 90 (212) 338-1547, http://home.ku.edu.tr/ aserpenguzel, http://microphotonics.ku.edu.tr, Koç University, Microphotonics Research Laboratory, Physics Department, Rumeli Feneri Yolu, Sariyer, Istanbul 34450 Turkey. The authors are with Koç University, Microphotonics Research Laboratory, Department of Physics, Rumeli Feneri Yolu, Sariyer, Istanbul 34450 Turkey. $\dagger$ Temel Bilici is also with Bogazici University, Department of Electrical and Electronics Engineering, Bebek, Istanbul 34342 Turkey. $\ddagger$ Senol Isci is also with Bogazici University, Biomedical Engineering Institute, Bebek, Istanbul 34342 Turkey. 


\section{MORPHOLOGY DEPENDENT RESONANCES}

The MDR's of the $\mu$-sphere can be explained as follows. As the light propagates around the inside surface of the $\mu$ sphere, it is confined by almost total internal reflection (TIR) ${ }^{8}$. After circumnavigating the $\mu$-sphere, the light wave returns to the starting point in phase to interfere constructively with itself. This constructive interference can occur only at certain discrete MDR wavelengths ${ }^{9}$. Each MDR is characterized by a mode number (n) and a mode order (l). Physically, (n) indicates the number of nodes in the internal intensity distribution as the polar angle is varied from $0^{\circ}$ to $180^{\circ}$. The mode order (1) indicates the number of nodes in the internal intensity distribution in the radial direction. For each set of mode numbers, there is a transverse electric (TE) and transverse magnetic (TM) MDR 8. For a given $\mu-$ sphere, the MDR occurs at specific value of the size parameter, $x_{n, l}$, which is given by $2 \pi a / \lambda_{n, l}$, where $\lambda_{n, l}$ is the light wavelength in vacuum and $\mathrm{a}$ is the radius of the $\mu$-sphere. These MDR's have been verified experimentally at optical wavelengths with micrometer-sized spheres.

The greatest impediment to the use of $\mu$-sphere resonators in practical devices has been the difficulty of efficiently light coupling into and out of the spheres. To couple light into or out of the $\mu$-sphere, it is necessary to overlap the evanescent field of the MDR with the evanescent field of the TIR. Such coupling has been implemented, by the use of thin tapered optical fibers, planar waveguide couplers, side-polished optical fibers, or high-index-prisms. If a $\mu$-sphere is placed near the angled surface, and within the evanescent field of the fiber-optic core, then there is an efficient energy exchange in resonance between the waveguide mode of the fiber and the MDR of the $\mu$-sphere ${ }^{10}$.

\section{EXPERIMENTAL SETUP}

A common approach is to polish down the cladding of an optical fiber to the point, where the evanescent field is locally exposed ${ }^{11}$. In the experiment, the OFHC is fabricated from a $810 \mathrm{~nm}$ single mode fiber with a core radius of $1.9 \mu \mathrm{m}$ and a refractive index of 1.462, and a cladding radius of $62.5 \mu \mathrm{m}$ with refractive index of 1.457 , which is laid on a glass substrate with a low curvature. The cladding of the fiber below the $\mu$-sphere is shaved down to $0.7 \mu \mathrm{m}$ in order to approach the core of the fiber. This excitation geometry effectively becomes the optical equivalent of a Gaussian beam with an infinite skirt length nassing near the II-snhere The transmission 1oss from the OFHC is corresnonding to $21 \mathrm{~dB}$ $(0.8 \%)$

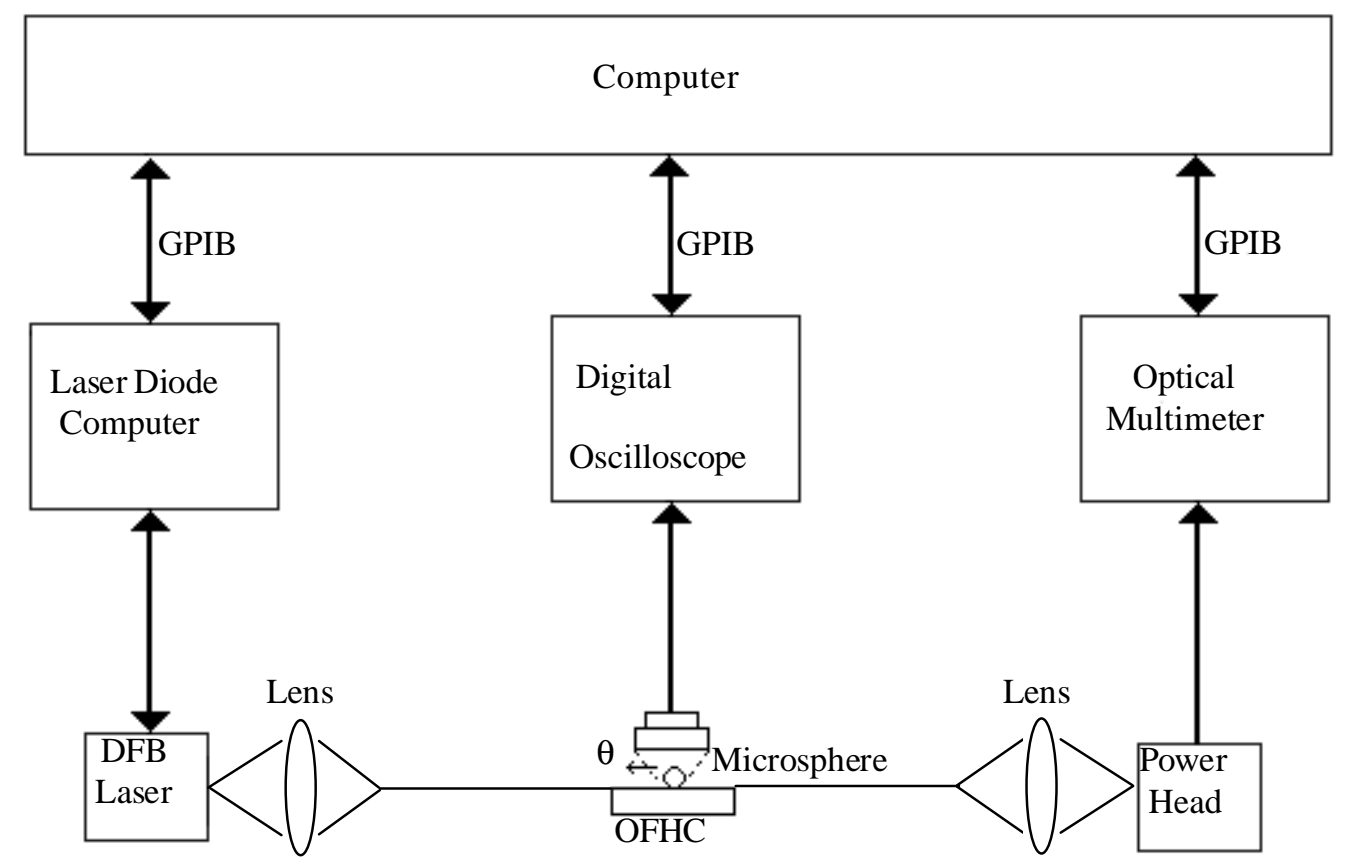

Figure 1. The schematic of the experimental setup of the optical channel dropping filter detector. 
A schematic of the experimental setup is shown in Fig. 1. The $\mu$-sphere used in the experiment is a BK7 type glass with a radius $\mathrm{a}=500 \mu \mathrm{m}$ and a refractive index of 1.502. MDR's of the $\mu$-sphere are excited by using a tunable distributed feedback (DFB) semiconductor laser. Wavelength tuning is achieved by tuning the temperature of the DFB with a laser diode controller. The elastically scattered polarization-independent light at $90^{\circ}$ is detected by using an unamplified windowless wide area $(4 \mathrm{~mm} \times 4 \mathrm{~mm})$ silicon photodiode placed approximately $1 \mathrm{~mm}$ away from the $\mu$-sphere-OFHC system as seen in Fig. 2. The photodetector signal is sent to a digital oscilloscope for monitoring and data acquisition. The optical power and the wavelength of the transmitted light are measured by an optical multimeter (OMM) with a silicon power/wave head. Device controls and data acquisition are performed with the IEEE-488 standard GPIB interface.

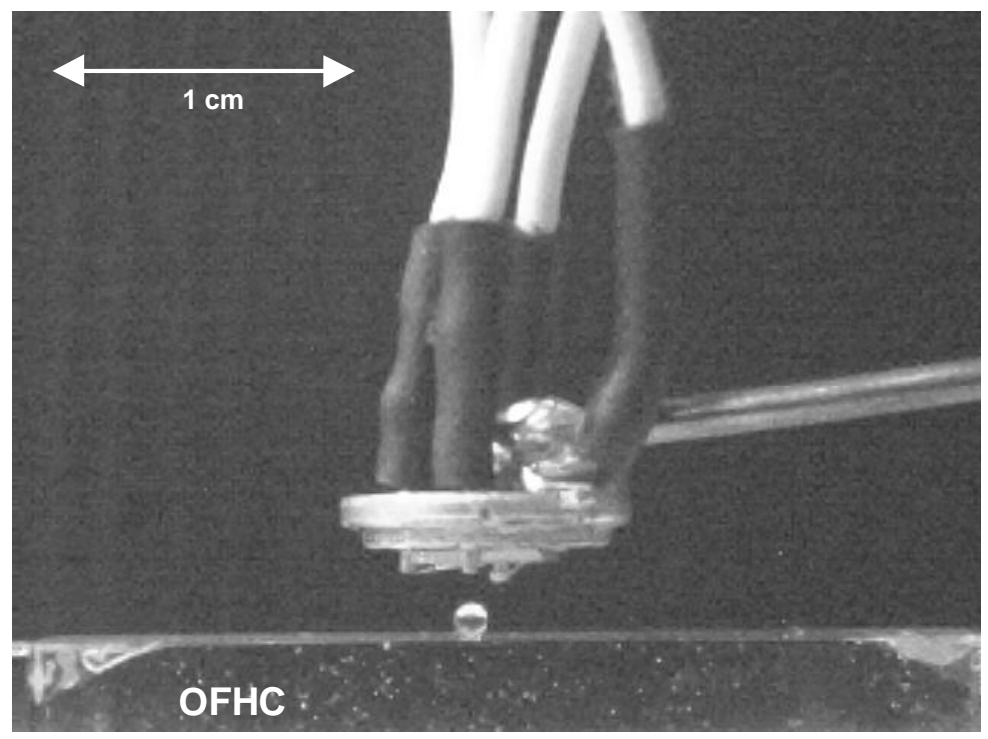

Figure 2. The side view picture of the optical fiber coupler, the microsphere, and the silicon detector.

\section{ELASTIC LIGHT SCATTERING}

Fig. 3 shows the elastic scattering and the power transmission spectra. MDR's in the elastic scattering and the associated dips in the transmitted power spectra are clearly observed. The temperature of the DFB laser is tuned between $19{ }^{\circ} \mathrm{C}$ and $30{ }^{\circ} \mathrm{C}$ at a constant current of $31.2 \mathrm{~mA}$. This temperature range corresponds to a wavelength range from $811.83 \mathrm{~nm}$ to $812.32 \mathrm{~nm}$. At the MDR wavelengths, the laser is coupled out of the fiber into the $\mu$-sphere, which results in dips in the transmitted signal. It is important to note that the fractional depth of the dips in the transmission spectra is not the same for all MDR's. This is due to different Q-factors and coupling efficiencies of the MDR's 10.

As shown in Fig. 4, the power coupled into MDR's was estimated from the power transmission spectra. For example, the MDR at $812.09 \mathrm{~nm}$ has a transmitted power of $67 \mu \mathrm{W}$. When the $\mu$-sphere is not present, the transmitted power is estimated to be approximately $75 \mu \mathrm{W}$. Therefore, the power coupled $\left(\mathbf{P}_{\mathbf{i}}\right)$ into the MDR is approximately $8 \mu \mathrm{W}$, which corresponds to a $0.5 \mathrm{~dB}(10 \%)$ loss in the transmission. For the experimental parameters of $\omega_{0}=2 \mu \mathrm{m}, \lambda=812.09 \mathrm{~nm}$, the elastically scattered intensity $\mathbf{I}_{\mathbf{i}}$ is calculated as $2 \mathrm{~kW} / \mathrm{cm}^{2}$ using $\mathrm{I}_{\mathrm{i}}=\mathrm{P}_{\mathrm{i}} / \sigma$, where the scattering cross section of the mode is $\sigma=\omega_{o} \lambda / \sqrt{2} \pi$ with the beam waist half width of $\omega_{0}$. Using the experimental parameters, the coupling efficiency of a Gaussian beam $(<\sigma\rangle)$ into MDR's is calculated as $\langle\sigma\rangle=\sqrt{2} \lambda / \pi^{2}$ and estimated to be $5.3 \%$ for the system, which correlates well with the measured $10 \%$ coupling efficiency 7 . Since the large area silicon photodiode has dimensions of $4 \times 4 \mathrm{~mm}$, the approximate power transfer efficiency of the $\mu$-sphere to the detector can be estimated as $(\theta / \pi) \times 10 \%=3.5 \%$, where $\theta$ is the angle covered by the photodiode (see Fig. 1 ). 


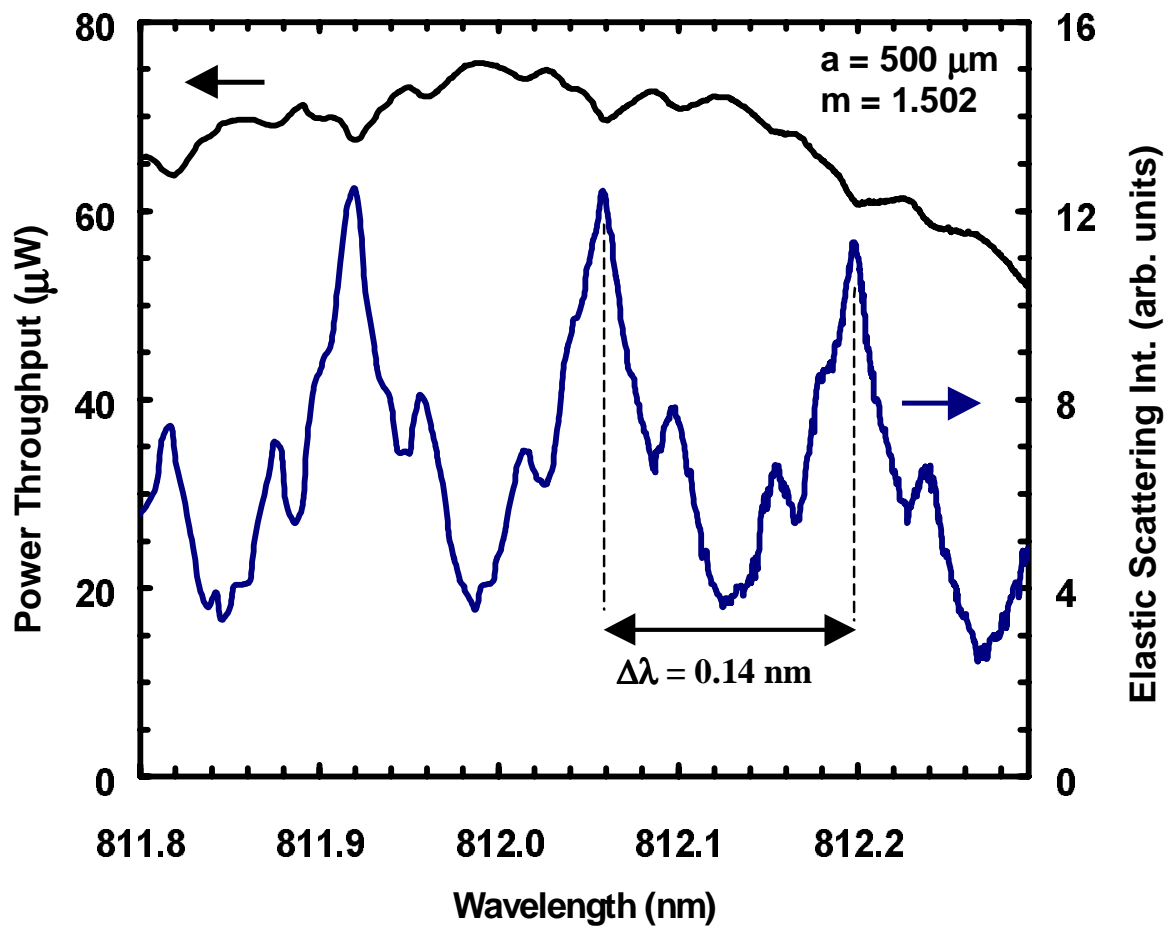

Figure 3. The low-resolution elastic scattering intensity and the power transmission spectra from the glass $\mu$ sphere.

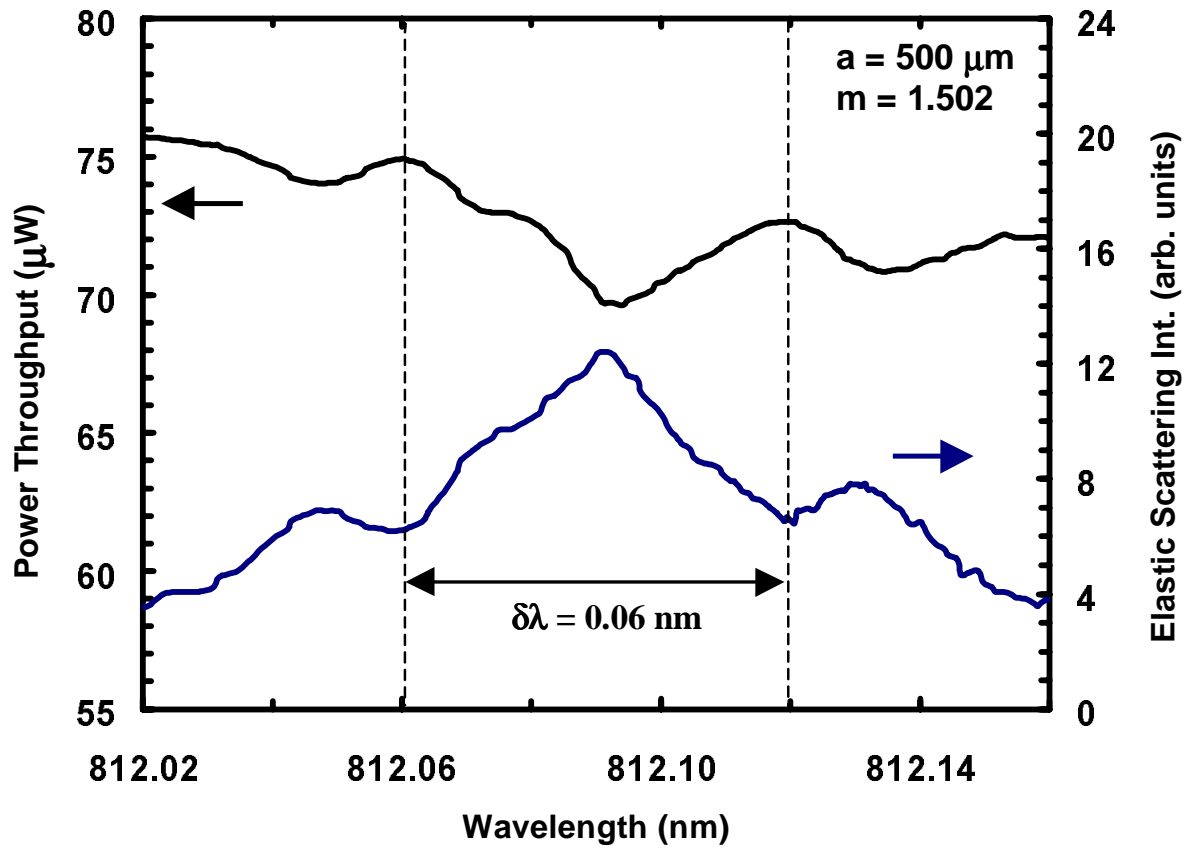

Figure 4. The high-resolution elastic scattering intensity and the power transmission spectra from the glass $\mu$ sphere. 
The scattered signal intensity increases with the decreasing the distance of the photodiode to the $\mu$-sphere. There is a background due to the OFC surface imperfections ${ }^{12}$. With a larger refractive index or smaller size $\mu$-sphere, the MDR peaks would be more clearly observable in the spectrum.

The bandwidths of the MDR's are measured to be $0.05 \mathrm{~nm}$ and the separation between the same mode order (l) MDR's with consecutive mode numbers $(\mathrm{n}), \Delta \lambda$, is measured to be about $0.14 \mathrm{~nm}$, which is consistent with the calculation based on the Mie scattering theory given by $\Delta \lambda=\left(\lambda^{2} \arctan \left(\mathrm{m}^{2}-1\right)^{1 / 2}\right) /\left(2 \pi \mathrm{a}\left(\mathrm{m}^{2}-1\right)^{1 / 2}\right)^{13}$.

The measured quality (Q) factor of the MDR's in the polarization-independent scattering spectrum is approximately of $10^{4}$. The high Q factor MDR's are superimposed upon a background of low Q-factor MDR's. Since no polarizer is used in the experiment, the spectrum gets contributions from both the TE and the TM modes, resulting in spectrally merged MDR's.

In practice, absorption, scattering on defects 2, scattering on impurities, residual surface roughness ${ }^{14}$ are responsible for the measured losses corresponding to Q-factors due to the presence of small shape distortions, and surface irregularities of the particle. The observed $Q$-values should be compared with the values determined by $Q^{-1}=Q_{a b s}{ }^{-1}+Q_{s}^{-1}+Q_{r}^{-1}$, where $\mathrm{Q}_{\mathrm{r}}^{-1}$ corresponds to the intrinsic diffraction leakage, and $\mathrm{Q}_{\mathrm{s}}^{-1}$ describes the losses due to light scattering by surface roughness and Rayleigh scattering inside the sphere. The optical absorption loss is expressed as $\mathrm{Q}_{\mathrm{abs}}{ }^{-1}=\alpha \lambda / 2 \pi \mathrm{m}$ with the absorption coefficient of $\alpha 3$. The presence of surface roughness and the molecular roughness would give higher-Q MDR's ${ }^{15}$, when the beam is aimed slightly inside the sphere's rim ${ }^{16}$.

\section{CONCLUSIONS}

In conclusion, detection of MDR's is achieved by using a silicon photodiode in close proximity to the $\mu$-sphere and OFHC. The OFHC, $\mu$-sphere, and photodiode system can be considered as a prototype of an integrated channeldropping filter detector. With the proper system design and optimization, it would be possible to totally drop the selected MDR's power from the transmission spectrum.

\section{ACKNOWLEDGMENTS}

We would like to acknowledge the partial support of this research by the Scientific and Technical Research Council of Turkey (TUBITAK) Grant No: TBAG-1952 and the European Office of Aerospace Research and Development (EOARD) Grants No: F61775-01-WE062 and FA8655-02-M-4086.

\section{REFERENCES}

1 S. M. Spillane, T. J. Kippenberg, and K. J. Vahala, "Ultralow-threshold Raman laser using a spherical dielectric microcavity", Nature, vol. 415, pp. 621-623, Feb. 2002.

2 V. Lefèvre-Seguin and S. Haroche, "Towards cavity-QED experiments with silica microspheres", Mat. Sci. Eng., vol. B48 pp. 53-58, 1997.

3 M. Kuwata-Gonokami and K. Takeda, "Polymer whispering gallery mode lasers", Opt. Mat., vol. 9, pp. 12-17, Jan. 1998.

4 V. S. Ilchenko, P. S. Volikov, V. L. Velichansky, F. Treussart, V. Lefèvre-Seguin, J.-M. Raimond, and S. Haroche, "Straintunable high-Q optical microsphere resonator", Optics Comm., vol.145, pp. 86-90, Jan. 1998.

5 M. Cai, G. Hunziker, and K. J. Vahala, "Fiber-optic add-drop device based on a silica microsphere-whispering gallery mode system," IEEE Photon. Technol. Lett., vol. 6, no. 6, pp. 686-687, June 1999.

6 H. C. Tapalian, J.-P. Laine, and P. A. Lane, "Thermooptical switches using coated microsphere resonators", IEEE Photon. Technol. Lett., vol. 14, no. 8, pp. 1118-1120, Aug. 2002.

7 A. Serpengüzel, S. Arnold, G. Griffel, and J. A. Lock, "Enhanced coupling to microsphere resonances with optical fibers," J. Opt. Soc. Am. B., vol. 14, no. 4, pp. 790-795, Apr. 1997.

8 M. Pelton and Y. Yamamoto, "Ultralow threshold laser using a single quantum dot and a microsphere cavity", Phys. Rev. A, vol. 59, no. 3, pp. 2418-2421, March 1999. 
9 B. R. Johnson, "Morphology-dependent resonances of a dielectric sphere on a conducting plane", J. Opt. Soc. Am. A, vol. 11, no. 7, pp. 2055-2064, July 1994.

10 J.-P. Laine, B. E. Little, and H. A. Haus, "Etch-Eroded Fiber coupler for whispering-gallery-mode excitation in high Q silica microspheres", IEEE Photon. Technol. Lett., vol. 11, no. 11, pp. 1429-1430, Nov. 1999.

11 O. Parrriaux, S. Gidon, and A. A. Kuznetsov, "Distributed coupling on polished single-mode optical fibers", Appl. Opt., vol. 20, no.14, pp. 2420-2423, July 1981.

12 A. Serpengüzel, S. Arnold, and G. Griffel, "Photonic atoms: Enhanced light coupling", in "Microcavities and photonic bandgaps: Physics and applications", J. Rarity and C. Weisbuch, Kluwer Academic Publishers, Netherlands, 1995.

13 R. Jia, D. Jiang, P. Tan, B. Sun, J. Zhang, and Y. Lin, "Photoluminescence study of $\mathrm{CdSe}_{\mathrm{x}} \mathrm{S}_{1-\mathrm{x}}$ quantum dots in a glass spherical microcavity", Chin. Phys. Lett., vol. 18, no. 10, pp. 1350-1352, 2001.

14 W. V. Klitzing, R. Long, V. S Ilchenko, J. Hare, and V. Lefèvre-Seguin, "Tunable whispering gallery modes for spectroscopy and CQED experiments", New J. Phys., vol. 3, pp. 14.1-14.14, Aug. 2001.

15 J. P. Barton, "Effects of surface perturbations on the quality and the focused-beam excitation of microsphere resonance", J. Opt. Soc. Am. A, vol. 16, no. 8, pp. 1974-1980, Aug. 1999.

16 H.-B. Lin, A. J. Campillo, and J. P. Barton, "Excitation localization principle for spherical microca vities", Opt. Lett., vol.23, no. 24, pp. 1921-1923, Dec. 1998. 